\title{
Vortex lattice in spin-imbalanced unitary Fermi gas
}

\author{
Jakub Kopyciński $\odot,{ }^{1,2}$ Wojciech R. Pudelko, ${ }^{1,3,4}$ and Gabriel Wlazłowski ${ }^{1,5, *}$ \\ ${ }^{1}$ Faculty of Physics, Warsaw University of Technology, Ulica Koszykowa 75, 00-662 Warsaw, Poland \\ ${ }^{2}$ Center for Theoretical Physics, Polish Academy of Sciences, Al. Lotników 32/46, 02-668 Warsaw, Poland \\ ${ }^{3}$ Swiss Light Source, Paul Scherrer Institut, CH-5232 Villigen PSI, Switzerland \\ ${ }^{4}$ Physik-Institut, Universität Zürich, Winterthurerstrasse 190, CH-8057 Zürich, Switzerland \\ ${ }^{5}$ Department of Physics, University of Washington, Seattle, Washington 98195-1560, USA
}

(Received 2 September 2021; accepted 5 November 2021; published 23 November 2021)

\begin{abstract}
We investigate the properties of a spin-imbalanced and rotating unitary Fermi gas. Using a density functional theory, we provide insight into states that emerge from a competition between Abrikosov lattice formation, spatial phase separation, and the emergence of the Fulde-Ferrell-Larkin-Ovchinnikov (FFLO) state. A confrontation of the experimental data [M. Zwierlein et al., Science 311, 492 (2006)] with theoretical predictions provides a remarkable qualitative agreement. In the case of gas confined in a harmonic trap, the phase separation into a superfluid core populated by the Abrikosov lattice and a spin-polarized corona is the dominant process. Changing confinement to a boxlike trap reverts the spatial location of the component: gas being in the normal state is surrounded by superfluid threaded by quantum vortices. The vortex lattice no longer exhibits the triangular symmetry, and the emergence of exotic geometries may be an indirect signature of the FFLO-like state formation in the system.
\end{abstract}

DOI: 10.1103/PhysRevA.104.053322

\section{INTRODUCTION}

The most fundamental technique for probing the superfluid properties of a system is via its response to rotation. This technique was used to demonstrate the superfluid character of a Bose-Einstein condensate by direct observation of the Abrikosov vortex lattice [1]. Later on, the same technique was applied to ultracold fermionic gases, providing direct evidence for the occurrence of the superfluidity over the entire BCS-BEC crossover [2]. Immediately, the focus was shifted toward the spin-imbalanced Fermi gas, where the principal mechanism responsible for the superfluidity, namely Cooper pairing, is modified. Theoretically, in spin-imbalanced systems the Fulde-Ferrell-Larkin-Ovchinnikov (FFLO) state should emerge [3-8]. The FFLO state corresponds to a ground state of the spin-imbalanced system, where the pairing between opposite spin partners with momentum $\boldsymbol{p}$ and $-\boldsymbol{p}+\boldsymbol{q}$ takes place. It manifests as the order parameter fluctuations in the form of $\Delta(\boldsymbol{r}) \sim e^{i \boldsymbol{q} \cdot \boldsymbol{r}}$ (FF) or $\Delta(\boldsymbol{r}) \sim \cos (\boldsymbol{q} \cdot \boldsymbol{r})$ (LO). In practical realization, these oscillation patterns are modified by the geometrical effects. In the case of experiments with ultracold atomic gases, the primary source of modifications comes from the trapping potential; see [9-11] for extensive overviews. In general, the trapping effect limits the FFLO state to be confined only in a limited volume. Moreover, it competes with the phase-separation phenomenon [12-16], i.e., the situation where the system separates spontaneously into a spin-symmetric superfluid part (the so-called BCS state) and a fully polarized state. In the case of the most popular

\footnotetext{
"gabriel.wlazlowski@pw.edu.pl
}

harmonic traps, the BCS superfluid occupies the trap center while the spatial fluctuations of the order parameter take place close to the system's boundary.

Recently, it has been predicted that the presence of the quantum vortices may stabilize the FFLO state in a wide range of control parameters [17]. This is because the vortices naturally introduce singularity lines $\Delta=0$ to the system. The population imbalance can be accommodated there $[3,18,19]$. Thus, locally around the line, the system can support conditions needed for the realization of the FFLO state, which in this case should manifest as the order parameter modulation as a function of distance from the vortex core. Moreover, the standard technique of generating vortices is due to the rotation of the trap. Then, the vortices localize typically close to the rotation axis, typically located in the trap center. In this way, the problem of the FFLO state generation close to the boundary can be suppressed. This simple picture is derived from studies of a single quantum vortex accompanied by the FFLO state. Unfortunately, presently available experimental measurements of the rotating spin-imbalanced Fermi gas [20] have not provided a clear signature of the FFLO state formation. Also, no indications of vortices with exotic internal structures were observed.

Superconductors, typically two-dimensional, are another class of materials where the FFLO state can be realized; see review paper [21]. In these systems, the FFLO state can be induced by applying an external magnetic field, which naturally favors the emergence of Abrikosov vortex states as well. These vortex states couple with the FFLO and make it difficult to identify them unambiguously. The difficulties in the interpretation triggered many studies targeted at a better understating of the coexistence of vortex lattice states and 
FFLO state. The studies include considerations of setups with a single vortex line [22-26] as well as the setups with vortex lattices [27-32]. Due to energetic reasons, the vortices tend to form triangular structures. At the same time, the nodal lines in the FFLO state prefer other geometries, where the most natural are square lattices emerging for $\Delta(x, y) \sim$ $\cos \left(q_{x} x\right) \cos \left(q_{y} y\right)$. The appearance of the vortex lattice of an exotic structure may be regarded as an indirect signature of the FFLO state emergence. This type of indirect protocol has also been suggested in the context of ultracold atomic gases [4].

In this paper, we provide results of numerical calculations for the spin-imbalanced Fermi gas being the subject of rotation by means of a density functional theory (DFT) [33]. It is a fully microscopic approach, designed for dealing with strongly interacting systems, with an explicit treatment of fermionic degrees of freedom. The rotating cloud of the unitary Fermi gas (UFG) is the subject of our studies. The interaction is tuned to the resonance $a k_{F} \rightarrow \infty$, where $a$ is the scattering length and $k_{F}=\left(3 \pi^{2} n\right)^{1 / 3}$ is the Fermi wave vector corresponding to the density $n$. The choice of the investigated system is motivated by the availability of the experimental data [20], which serves here as a reference point for comparing with theoretical predictions. The uniform unitary gas, similarly to the free Fermi gas, is characterized only by a single scale: average interparticle distance $n^{1 / 3} \sim k_{F}$. This property significantly limits the possible forms of an energy density functional (EDF), and it allows for the successful formulation of the DFT framework for the UFG - the approach being in principle exact; see for a review [33]. The framework has been already applied for studies of quantum vortices [34-37], revealing a remarkable agreement with experiment [38]. Also, it admits the development of the FFLO state at unitarity [39]. Applying the DFT framework to the system that combines vortices and spin-polarization effects will serve as the next step of the validation process of the theory. Note that the spin-imbalance effects are beyond the reach of simplified methods that were used so far for studies of vortex lattices, like the Gross-Pitaevskii equation or the local phase density approximation to the Bogoliubov-de Gennes equations [40]. In the Bose-Einstein condensate limit it is possible to describe the system by a set of coupled equations for composite bosons and excess fermions [41].

These studies are also relevant for neutron stars, particularly for magnetars $[42,43]$. They are assumed to be superfluid and filled with a large number of vortices. The observed rapid change of rotation frequency, called the neutron star glitch, is regarded as a direct manifestation of the superfluid character of the star interior [44-47]. Moreover, a strong magnetic field of the order of or larger than $10^{16} \mathrm{G}$ is expected to be present inside the magnetars. It can effectively spin-polarize the neutron matter and induce unconventional superfluid phases, including FFLO [48-50]. Whether the FFLO state can develop in the rotating star remains an unanswered question. The only methodology that can provide insight into this problem is through numerical simulation. Presently, the DFT method has become one of the standard tools for microscopic studies of nuclear systems as well [51-53], and in principle, it can also be applied to rotating and spin-polarized neutron matter. However, a prior validation of the framework by applying it to strongly interacting terrestrial systems under similar conditions is desired. The ultracold atomic setup, as discussed here, suits this purpose very well.

\section{FRAMEWORK}

In the calculations, we use the density functional theory. The chosen energy density functional is known as asymmetric superfluid local density approximation (ASLDA), designed specifically for the strongly interacting Fermi gas at unitarity [33]. It has the following form (we use metric system $m=$ $\left.\hbar=k_{B}=1\right)$ :

$$
\begin{aligned}
\mathcal{E}_{\mathrm{aslda}}= & \sum_{\sigma=\{\uparrow, \downarrow\}} \frac{\alpha_{\sigma}(p) \tau_{\sigma}}{2}+\beta(p)\left(n_{\uparrow}+n_{\downarrow}\right)^{\frac{5}{3}} \\
& +\gamma(p) \frac{\nu^{\dagger} v}{\left(n_{\uparrow}+n_{\downarrow}\right)^{\frac{1}{3}}}+\sum_{\sigma=\{\uparrow, \downarrow\}}\left[1-\alpha_{\sigma}(p)\right] \frac{\boldsymbol{j}_{\sigma}^{2}}{2 n_{\sigma}} .
\end{aligned}
$$

This functional consists of the kinetic term, normal and pairing interaction terms, and a term responsible for restoring the Galilean invariance, respectively. These are functions of particle density $n_{\sigma}$, the kinetic density $\tau_{\sigma}$, the anomalous density $\nu$, and the probability current $\boldsymbol{j}_{\sigma}$, where $\sigma=\{\uparrow, \downarrow\}$ indicates the spin components. The densities and currents are parametrized via an orthonormal set of Bogoliubov quasiparticle wave functions $\left\{u_{n, \uparrow}(\boldsymbol{r}), u_{n, \downarrow}(\boldsymbol{r}), v_{n, \uparrow}(\boldsymbol{r}), v_{n, \downarrow}(\boldsymbol{r})\right\}$ as follows:

$$
\begin{gathered}
n_{\sigma}(\boldsymbol{r})=\sum_{\left|E_{n}\right|<E_{c}}\left|v_{n, \sigma}(\boldsymbol{r})\right|^{2} f_{\beta}\left(-E_{n}\right), \\
\tau_{\sigma}(\boldsymbol{r})=\sum_{\left|E_{n}\right|<E_{c}}\left|\nabla v_{n, \sigma}(\boldsymbol{r})\right|^{2} f_{\beta}\left(-E_{n}\right), \\
v(\boldsymbol{r})=\sum_{\left|E_{n}\right|<E_{c}} u_{n, \uparrow}(\boldsymbol{r}) v_{n, \downarrow}^{*}(\boldsymbol{r}) \frac{f_{\beta}\left(-E_{n}\right)-f_{\beta}\left(E_{n}\right)}{2}, \\
\boldsymbol{j}_{\sigma}(\boldsymbol{r})=\sum_{\left|E_{n}\right|<E_{c}} \operatorname{Im}\left[v_{n, \sigma}(\boldsymbol{r}) \nabla v_{n, \sigma}^{*}(\boldsymbol{r})\right] f_{\beta}\left(-E_{n}\right) .
\end{gathered}
$$

These wave functions have the interpretation that $\left|u_{n, \sigma}(\boldsymbol{r})\right|^{2}$ is the probability density of the $n$th state being occupied with a spin $\sigma$ hole and $\left|v_{n, \sigma}(\boldsymbol{r})\right|^{2}$ with a spin $\sigma$ particle. The FermiDirac distribution $f_{\beta}(E)=1 /[\exp (\beta E)+1]$ is introduced in order to model temperature $k_{B} T=\beta^{-1}$ effects. To avoid divergences, only states with quasiparticle energies smaller than the cutoff energy $E_{c}$ are considered and the regularization scheme as described in $[33,54]$ is applied. The coupling constants $\alpha_{\sigma}(p), \beta(p)$, and $\gamma_{\sigma}(p)$ are functions of the local spin polarization of the system $p(\boldsymbol{r})=\frac{n_{\uparrow}(\boldsymbol{r})-n_{\downarrow}(\boldsymbol{r})}{n_{\uparrow}(\boldsymbol{r})+n_{\downarrow}(\boldsymbol{r})}$. They are represented as polynomials, and fitted to the quantum Monte Carlo calculations for spin-symmetric as well as spin-imbalanced systems [33].

Equations of motion are determined by minimization of the functional

$$
\begin{aligned}
E= & \int\left[\mathcal{E}_{\text {aslda }}(\boldsymbol{r})+V_{\text {ext }}(\boldsymbol{r}) n(\boldsymbol{r})\right] d \boldsymbol{r} \\
& -\mu_{\uparrow} N_{\uparrow}-\mu_{\downarrow} N_{\downarrow}-\Omega_{z} L_{z},
\end{aligned}
$$

where $V_{\text {ext }}$ is the external potential that couples to the total density $n=n_{\uparrow}+n_{\downarrow}, \mu_{\sigma}$ are chemical potentials (Lagrange multipliers) that control particle number $N_{\sigma}=\int n_{\sigma}(\boldsymbol{r}) d \boldsymbol{r}$, and 
$\Omega_{z}$ is angular frequency that couples to $z$ th component of the angular momentum $\boldsymbol{L}=\int \boldsymbol{r} \times\left(\boldsymbol{j}_{\uparrow}+\boldsymbol{j}_{\downarrow}\right) d \boldsymbol{r}$. The last term is equivalent to the transformation of the problem to a rotating frame.

The minimization with respect to the quasiparticle orbitals provides equations that formally have the structure of Bogoliubov-de Gennes equations:

$$
\begin{gathered}
\left(\begin{array}{cc}
h_{\uparrow}-\mu_{\uparrow}-\Omega_{z} l_{z} & \Delta \\
\Delta^{*} & -\left(h_{\downarrow}-\mu_{\downarrow}-\Omega_{z} l_{z}\right)^{*}
\end{array}\right)\left(\begin{array}{c}
u_{n, \uparrow} \\
v_{n, \downarrow}
\end{array}\right) \\
=E_{n}\left(\begin{array}{c}
u_{n, \uparrow} \\
v_{n, \downarrow}
\end{array}\right),
\end{gathered}
$$

with $h$ being the single-particle Hamiltonian

$$
h_{\sigma}=-\frac{1}{2} \nabla \alpha_{\sigma} \nabla+\frac{\delta \mathcal{E}_{\text {aslda }}}{\delta n_{\sigma}}+V_{\text {ext }}-\frac{i}{2}\left\{\frac{\delta \mathcal{E}_{\text {aslda }}}{\delta \boldsymbol{j}_{\sigma}}, \nabla\right\}
$$

and $\Delta=-\frac{\delta \mathcal{E}_{\text {aslda }}}{\delta \nu^{*}}$ being the pairing field, which plays the role of the superfluid order parameter. The single-particle angular momentum operator is $l_{z}=-i\left(x \frac{\partial}{\partial y}-y \frac{\partial}{\partial x}\right)$. The remaining components of the quasiparticle wave functions $v_{n, \uparrow}$ and $u_{n, \downarrow}$ are obtained by using the symmetry relation: $u_{n, \uparrow} \mapsto v_{n, \uparrow}^{*}$, $v_{n, \downarrow} \mapsto u_{n, \downarrow}^{*}$, and $E_{n} \mapsto-E_{n}$.

The simulations consider the following scenarios: the nonrotating gas trapped in a harmonic potential and the rotating gas in a harmonic and in a box trap. In all cases, we assume that the trapping acts only in the $x$ and $y$ directions, while in the $z$ direction the system is uniform. For example, the harmonic potential has the form of $V_{\text {ho }}(\boldsymbol{r})=\frac{1}{2} m \omega^{2} \rho^{2}$, where $\rho=\sqrt{x^{2}+y^{2}}$. This simplification allows us to consider systems with a number of vortices $\sim 50$, which is the same order as observed in the experiment. In the case of the box potential, we assumed that the system is kept in a tube of radius $R_{t}$. Precisely, the potential is zero for $\rho<R_{t}$, and next within interval $\Delta R_{t} \ll R_{t}$ it smoothly rises to constant value $4 \varepsilon_{F}$, where $\varepsilon_{F}=k_{F}^{2} / 2$ is the Fermi energy. In each scenario, we keep the total number of atoms $N=N_{\uparrow}+N_{\downarrow}$ fixed, and we vary the spin imbalance $\delta=\left(N_{\uparrow}-N_{\downarrow}\right) / N$. The set of considered imbalances is the following: $\delta=0,5,10,18,28,39,50,62,80$, and 90 percent (the $5 \%$ value is for the gas in the box potential only).

The simulations were executed on a three-dimensional spatial mesh of size $N_{x} \times N_{y} \times N_{z}$, and periodic boundary conditions are assumed. The lattice spacing was selected to be $d x \approx \xi_{\mathrm{BCS}}$, where $\xi_{\mathrm{BCS}}=k_{F} / \pi \Delta$ is the BCS coherence length, which defines the expected vortex size. Here, as the Fermi momentum $k_{F}=\left(3 \pi^{2} n\right)^{1 / 3}$ and the pairing field $\Delta$ we used values corresponding to the center of the trap. The system temperature is close to absolute zero and reads $k_{B} T=$ $0.01 \varepsilon_{F}$. The angular frequency of the harmonic trap was set to $\omega / \varepsilon_{F}=33.7 \times 10^{-3}$, and $R_{t}=56 \xi_{\mathrm{BCS}}$ for the box potential. When considering the rotating systems, we used $\omega / \Omega_{z}=$ $\frac{110}{70}$, which matches the ratio used in the experiment [20]. The simulation parameters are summarized in Table I. Equation (7) was solved self-consistently, and the Broyden algorithm was applied to improve the convergence rate $[55,56]$. The calculations were executed with the use of W-SLDA Toolkit [36,38,57].
TABLE I. Parameters used in simulations: number of atoms $N$,

\begin{tabular}{|c|c|c|c|c|}
\hline$N$ & $\omega / \varepsilon_{F}$ & $\Omega_{z} / \varepsilon_{F}$ & Lattice size & $d x / \xi_{\mathrm{BCS}}$ \\
\hline \multicolumn{5}{|c|}{ Nonrotating case in the harmonic potential } \\
\hline 16324 & $33.7 \times 10^{-3}$ & 0 & $96^{2} \times 48$ & 1.33 \\
\hline \multicolumn{5}{|c|}{ Rotating case in the harmonic potential } \\
\hline 8162 & $33.7 \times 10^{-3}$ & $21.7 \times 10^{-3}$ & $128^{2} \times 32$ & 1 \\
\hline \multicolumn{5}{|c|}{ Rotating case in the box potential } \\
\hline 34032 & $\mathrm{~N} / \mathrm{A}$ & $21.7 \times 10^{-3}$ & $128^{2} \times 32$ & 1 \\
\hline
\end{tabular}
harmonic trap angular frequency $\omega$, rotation angular frequency $\Omega_{z}$, lattice size and spacing $d x$.

\section{HARMONIC OSCILLATOR TRAP}

\section{A. Ground state properties}

Before we start to consider the system in the rotating frame, we analyze ground states as a functions of the spin polarization $\delta$ revealed by the ASLDA method. The simulated density profiles are shown in Fig. 1(a). First of all, we observe the phase separation there. The system spontaneously splits into the spin-polarized corona and the superfluid core. Hereafter, we define the polarized region as a volume where $p(\boldsymbol{r})>p_{\text {crit }}=50 \%$. We find that this definition reasonably well selects the region where $\Delta(\boldsymbol{r}) \approx 0$. The advantage of using it is that it is insensitive to the presence of quantum vortices and other singularity lines, where $\Delta=0$ by definition. In Fig. 1(a) the light gray center means there is a condensate with a high particle density. The darker space corresponds to unpaired particles. In the minority component, it is clearly seen for which population imbalance the condensation occurs. For $\delta=80 \%$ and $90 \%$, the dense part is no longer visible, so the condensation vanishes there.

Our attention should be drawn to the visible rings with a higher density (for $\delta>0 \%$, most clearly seen for $\delta=$ $62 \%$ ). The positions of the majority component density local maxima $n_{\uparrow}$ agree with the local minima positions of the minority component density $n_{\downarrow}$ and the other way round-when $n_{\uparrow}$ reaches a local minimum, $n_{\downarrow}$ takes a locally maximal value. The densities oscillate on the core-corona interface, and they are directly linked to oscillations of the order parameter $\Delta(\boldsymbol{r})$. The number of oscillations ranges from 1 to 5 for the imbalances from $\delta=10 \%$ to $62 \%$. We can see this in Fig. 2 . The order parameter vanishes for $\delta \gtrsim \delta_{\text {crit }} \approx 80 \%$. This is evidence that the fermionic condensate is no longer found there. The obtained $\delta_{\text {crit }}$ agrees well with experimentally measured value, which is located in the range $75 \%-80 \%[13,16,58,59]$. Contrary to the simulation, the oscillations of the density have not been detected so far experimentally. It needs to be emphasized that our geometry corresponds to the case of an extremely elongated trap in the $z$ direction. It is known that increasing the elongation of the trap also increases the strength of the oscillations $[11,60]$. Thus, we expect that this particular feature is overestimated in our calculations in comparison to experimental realization.

The oscillating order parameter is typically regarded as a signature of the FFLO state. However, proximity effects in the superconductor-ferromagnet (SF) can also induce the 


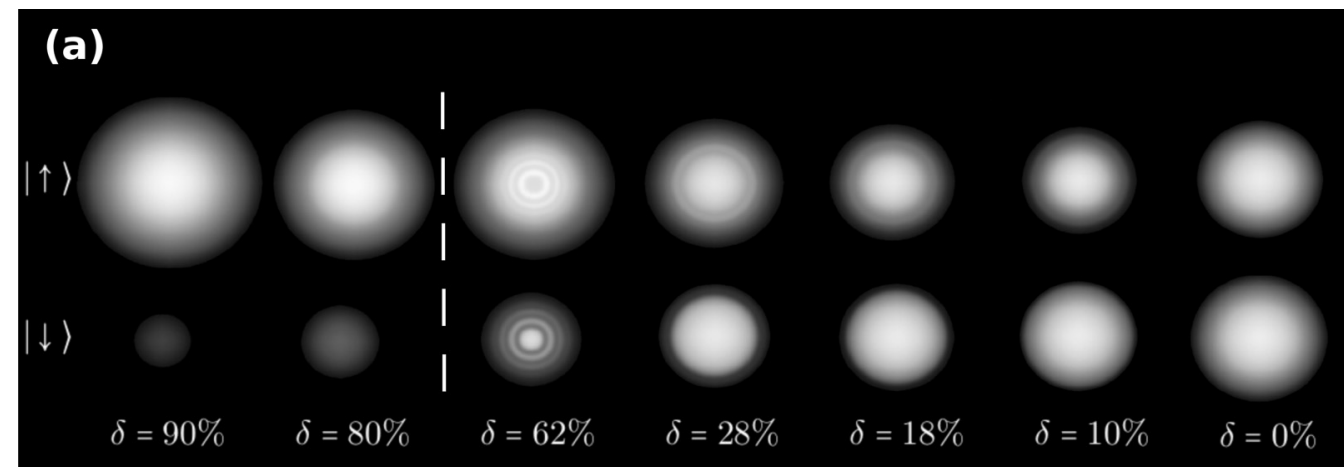

(b)

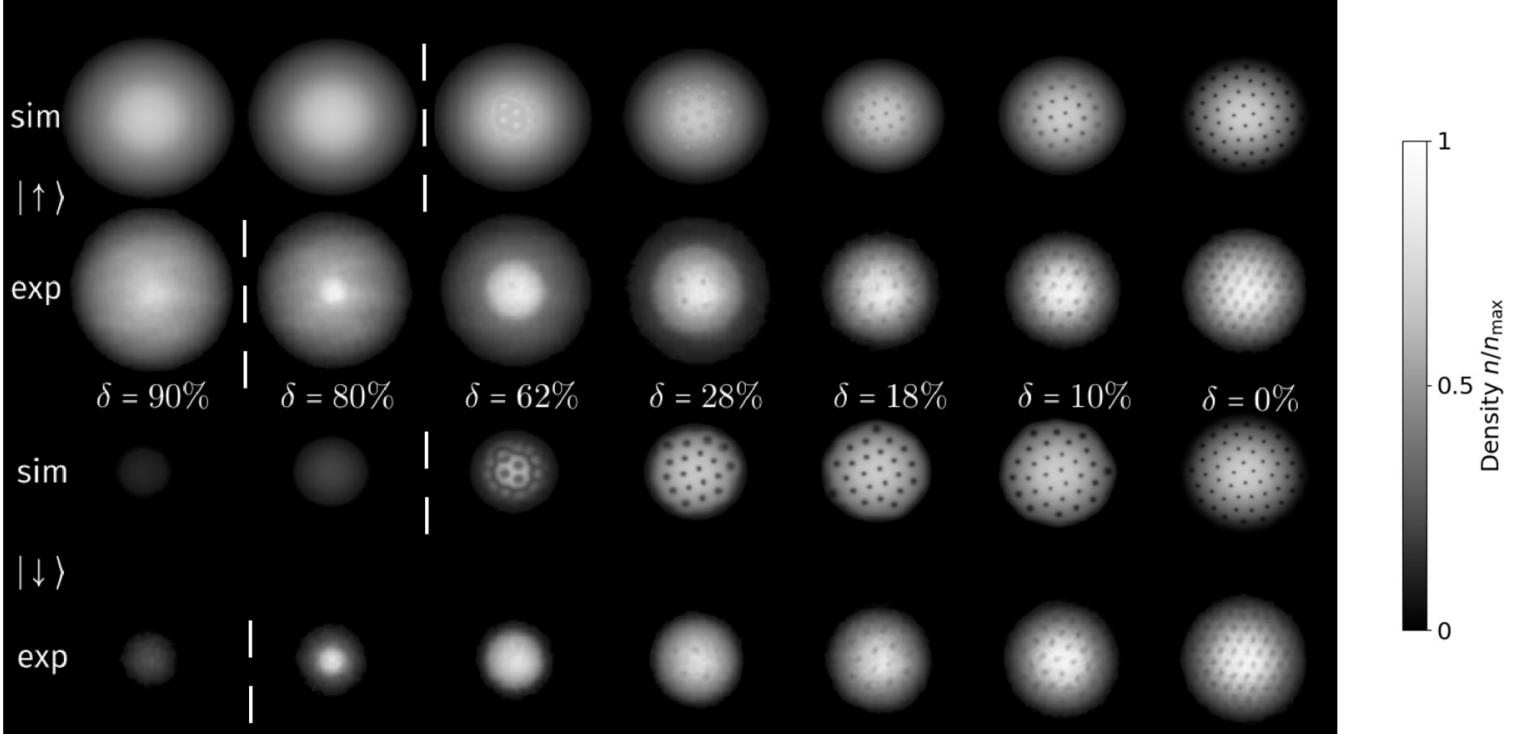

(c)

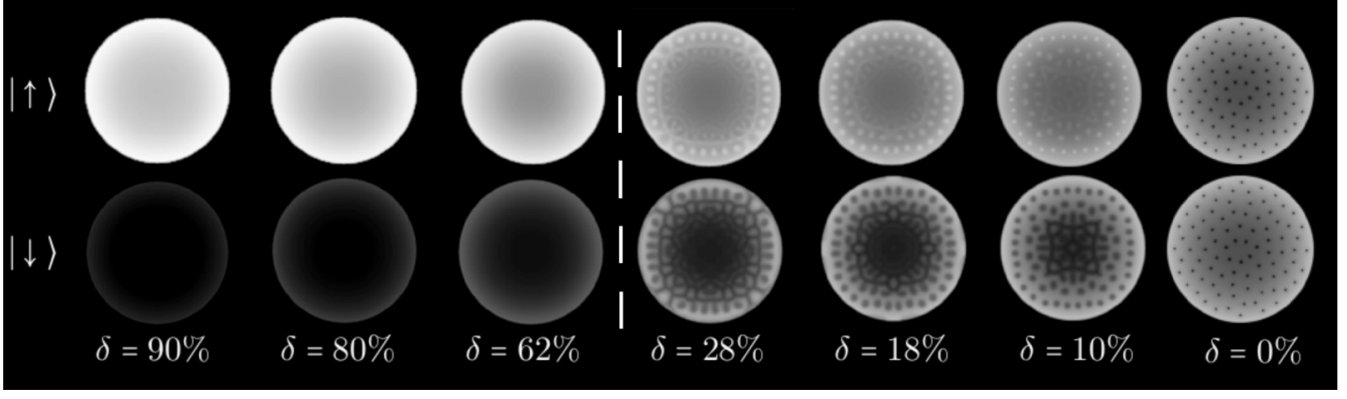

FIG. 1. (a) Simulated densities $n(x, y, z=0)$ for a nonrotating system, trapped in a harmonic potential, separately for the majority $|\uparrow\rangle$ and minority $|\downarrow\rangle$ components and different population imbalances $\delta$. (b) Comparison between the experimental absorption images (exp) and the simulated densities $n(x, y, z=0)(\operatorname{sim})$ for a rotating system trapped in a harmonic potential, separately for the majority $|\uparrow\rangle$ and minority $|\downarrow\rangle$ components and different population imbalances $\delta$. Experimental figures taken from Ref. [20] and reprinted with permission from AAAS. (c) Simulated densities $n(x, y, z=0)$ for a rotating system, trapped in a box potential, separately for the majority $|\uparrow\rangle$ and minority $|\downarrow\rangle$ components and different population imbalances $\delta$. The dashed line sets (on the right side of it) the cases with the superfluid phase apart from the ones (on the left) without it. In panel (b), we can see the discrepancy in the position of this line as the experimental absorption image was taken for $\frac{1}{k_{F} a}=0.2$ (i.e., slightly on the BEC side).

oscillations. They are typically well described by [61]

$$
\Delta(r) \propto \frac{1}{r-r_{0}} \exp \left(-\frac{r-r_{0}}{\xi_{1}}\right) \cos \left(\frac{r-r_{0}}{\xi_{2}}\right) .
$$

The two characteristic length scales are the correlation decay length $\xi_{1}$ and oscillating length $\xi_{2} ; r_{0}$ is the location of the superfluid-ferromagnet transition. Empirically, we find that the simulated data can be well fitted to the function (9); see Fig. 2. For a spin-balanced system we set $\xi_{2} \rightarrow \infty$. Then it is an analog of a superconductor-normal junction. It is expected to be the case, since at the edges of the cloud $T / \varepsilon_{F} \gg 1$, and effectively the gas is in the normal state there. In the case of the FFLO state, the oscillation length is expected to be related to mismatch of Fermi wave vectors related to each 


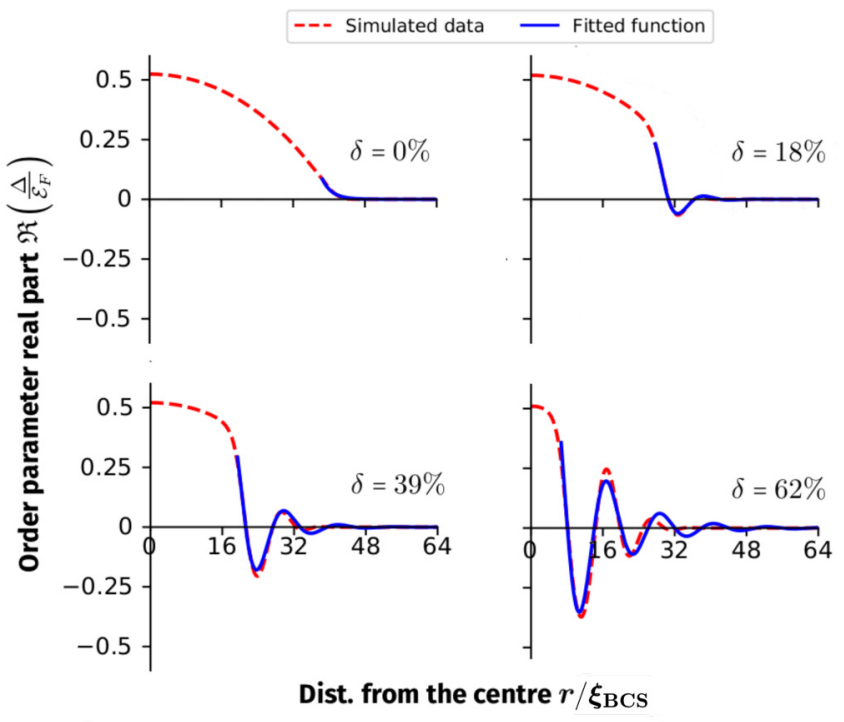

FIG. 2. Real part of the order parameter $\Delta$ as a function of the distance from the system center for different population imbalances [dashed (red) line]. Fit to Eq. (9) is shown by solid (blue) line.

spin component $\xi_{2}^{-1} \sim k_{F, \uparrow}-k_{F, \downarrow}$, and should decrease as we increase $\delta$. Unfortunately, the obtained value from the fit is fairly constant $k_{F} \xi_{2} \approx 2.77$. For this reason, we conclude that for a nonrotating setup, phase separation is a dominant process.

\section{B. System with rotation}

We start an analysis of results for the systems with rotation by comparing density sections $n_{\sigma}(x, y, z=0)$ with the absorption images taken experimentally [20]. This is done in Fig. 1(b). It needs to be emphasized that the experimental measurements are done after ramping the magnetic field to the Bose-Einstein condensate (BEC) regime and releasing the gas from the trap. The target value of the magnetic field, rate of the ramping, as well as expansion time after the release may impact the configuration in the final state $[62,63]$. Moreover, the noninteracting gas in the corona expands differently from the interacting core [64]. Here we do not account for all these effects related to the experimental measurement-we directly compare numerical density profiles at unitarity with the experimental signal measured after sweeping to the molecular regime. We find that, in general, the ASLDA simulation results are qualitatively consistent with the experiment. It is quite remarkable since we do not make any attempts to match the experimental results (there are no free parameters in the theory that we fit to the experiment). Most likely, the rigidity of the order parameter with respect to the fast sweeps of the magnetic field is responsible for overall consistency between the experiment and the simulation [65].

The ASLDA correctly reproduces the gross properties of the emerging vortex lattices (see also Fig. 7). For the population imbalances, where we see the superfluid fraction $(\delta$ from 0 to $62 \%$ ), the vortices appear. This is in agreement with the experiment where critical population imbalance for rotating case was reported as $\delta_{c} \approx 70 \%$. Some unpaired particles do not leave the superfluid part and tend to occupy the vortices inside. This is visible when comparing the majority and mi- nority component densities. We see that vortices (especially those outside the superfluid core) are filled with the spin-up particles, while the opposite spin component exhibits suppression in the core. This is due to the specific occupation of Andreev states localized in the vortex cores $[3,18,19]$. Experimental images do not reveal such structure of the vortex cores. Instead, both spin components are suppressed in the core, irrespective of the population imbalance. To date, there are no theoretical studies that investigate the impact of the magnetic field sweep to the BEC side of the resonance on the vortex structure. Technically, this procedure is needed to visualize vortices, which otherwise are not detected directly at unitarity. The imaging operation (sweep and expansion) takes about $\Delta t_{\text {img }} \sim 10^{3} \hbar / \varepsilon_{F}$. On the other hand, the time needed by an atom at the Fermi surface to travel distance of the order of vortex core size is $\Delta t_{\xi} \sim 1 \hbar / \varepsilon_{F}$. Clearly, till imagining there is sufficient time for vortices to change their internal structure. Similar argumentation also applies to particles trapped in the vicinity of nodal lines associated with the FFLO state. In the case of slow ramps from the resonance to the molecular limit, it can be assumed that the process is adiabatic and then the results of static calculations as presented in [66] may be applicable. In such a case information about the initial vortex structure will be also lost.

The detailed analysis of the vortex lattices in terms of the densities, polarization, and the superfluid core radii, for two selected population imbalances, is shown in Fig. 3. We can distinguish two types of regions that accumulate the spin polarization: vortex cores and, as for the case without rotation, the edge of the cloud. This can be understood based on energetic considerations: unpaired particles tend to accumulate in regions where $\Delta \approx 0$ and do not break Cooper pairs in the surrounding superfluid. The superfluid radii (volume region where $\Delta>0$ ) are larger here than in the nonrotating case. On average, the radius increases by $16.5 \%$ with the standard deviation of $4.8 \%$. This enlargement is caused by centrifugal force. For the population imbalances $\delta=80 \%$ and $90 \%$, the superfluidity disappears.

For low population imbalances, vortices tend to organize into the triangular lattice in the superfluid core, while perturbations in the ordering emerge mainly close to the boundaries. For $\delta=39 \%, 50 \%$, and $62 \%$, there are density oscillations also resulting in the oscillating polarization. They are correlated with the order parameter fluctuations, as in the case of the superfluid-ferromagnet proximity effect. Precisely, the oscillations are accompanied with additional jumps of phase by $\pi$; see case $\delta=50 \%$ in Fig. 3. This indicates that the FFLO-type phase may coexist with the vortex lattice for high population imbalances and, certainly, smaller than the critical population imbalance $\delta_{c}$.

We have not identified any signatures of quantum vortices hosting inside the FFLO state, as suggested in [17]. Instead, we find emergence of vortices with reversed circulation, predicted in [19]. To demonstrate this, we consider the current profile $\boldsymbol{j}_{\sigma}(\boldsymbol{r})$. In general the current is dominated by overall rotation of the system with velocity $\boldsymbol{v}^{(\Omega)}(\boldsymbol{r})=\boldsymbol{\Omega} \times \boldsymbol{r}$, where $\boldsymbol{\Omega}=\left[0,0, \Omega_{z}\right]$ (see also Fig. 6). Thus, to extract current within the rotating frame we apply transformation $\boldsymbol{j}_{\sigma}^{\prime}=$ $\boldsymbol{j}_{\sigma}-n_{\sigma} \boldsymbol{v}^{(\Omega)}$. The current $\boldsymbol{j}_{\sigma}^{\prime}$ acquires significant values only in the vicinity of quantum vortices. In Fig. 4 we show this for 
(a)

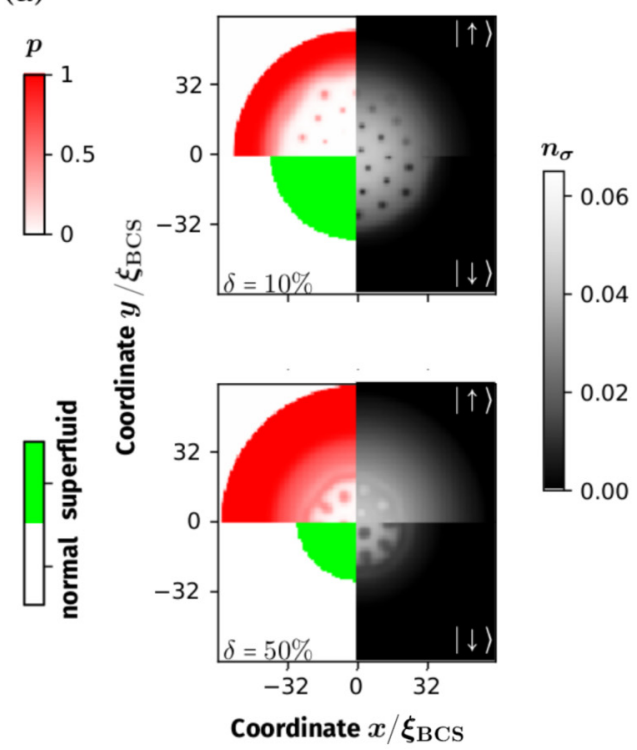

(b)

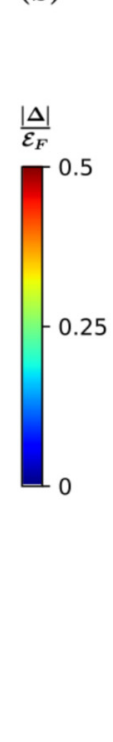

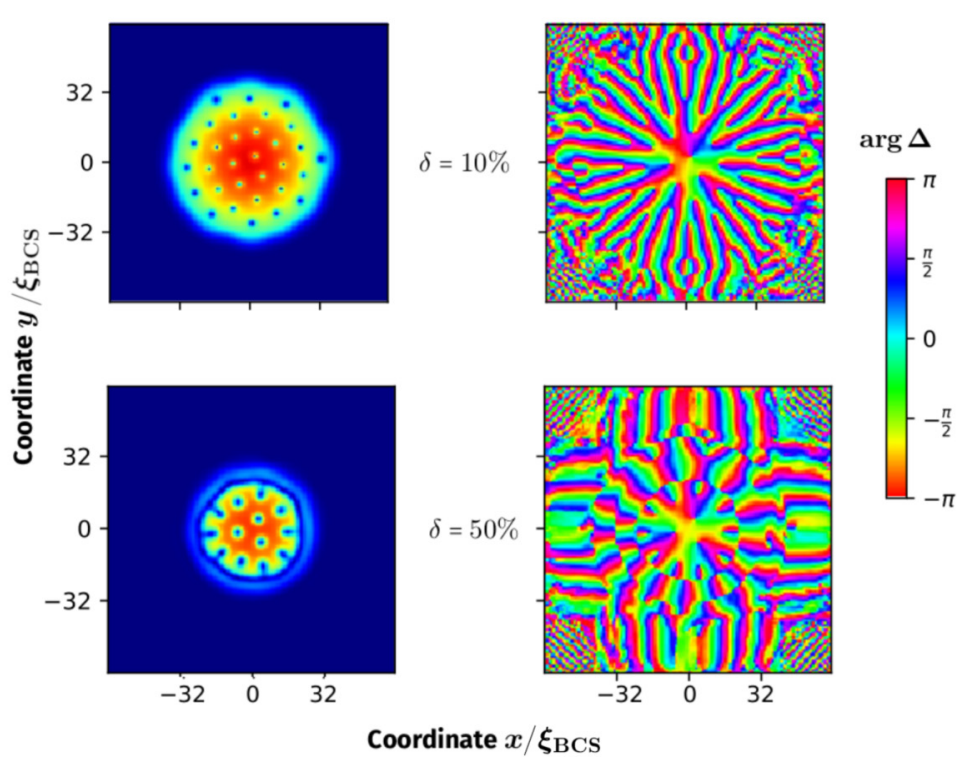

FIG. 3. (a) Polarization $p(x, y, z=0)$ (top left part of each subplot), densities $n_{\sigma}(x, y, z=0)$ of the majority (top right part of each subplot), and the minority components (bottom right part of each subplot), as well as the indication of the superfluid region (bottom left part of each subplot). (b) Pairing gap absolute value $|\Delta(x, y, z=0)|$ (left column) and its phase $\arg \Delta(x, y, z=0)$ (right column). The rows correspond to $\delta=10 \%$ (top) and $50 \%$ (bottom).

representative vortices for a few selected population imbalances. In the systems with a small population imbalance such as $\delta=0$ or $18 \%$, the total current in the rotating frame is moving counterclockwise around the vortex. This situation changes, when we increase the imbalance. In the case of $\delta=$ $50 \%$ we may notice there is a small region inside the vortex, where the current is rotating clockwise. For the total current in the system with $\delta=62 \%$ this effect is considerably more visible. Clearly, in the vortex lattices with high population imbalance we can find spin-polarized vortices with reversed flow.

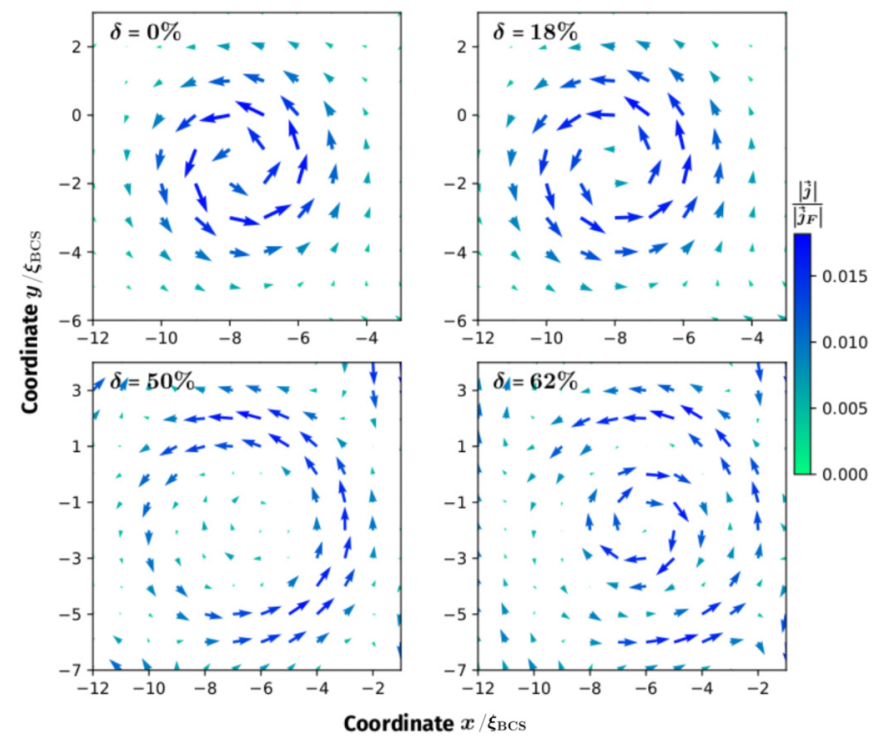

FIG. 4. Probability current (total $\boldsymbol{j}^{\prime}=\boldsymbol{j}_{\uparrow}^{\prime}+\boldsymbol{j}_{\downarrow}^{\prime} \quad$ for $\delta=0 \%, 18 \%, 62 \%$, minority component $j_{\downarrow}^{\prime}$ for $\delta=50 \%$ ) in a rotating frame. Only the surroundings of selected vortices are presented. The reversed flow is visible for $\delta=50 \%$ and $62 \%$.

\section{IMPACT OF THE TRAPPING POTENTIAL GEOMETRY}

The new generation of experiments with 3D ultracold gases utilize typically boxlike traps [67-71]. In this section we provide predictions of the ASLDA method in the case of such a trap. The gas in the nonrotating box trap is uniform (except regions in close vicinity of boundaries). However, once we introduce rotation, we observe a fundamental change in the system. Neither the density maximum is placed in the system center, nor vortices create a triangular lattice, except the case $\delta=0 \%$, where vortices tend to organize in the Abrikosov lattice. The lack of a perfect simulated Abrikosov lattice for the spin-symmetric system can be due to boundary effects; however, we cannot exclude that the algorithm is stuck in a metastable stable state (although we did a few tries with different starting points). Due to the centrifugal force, the particles are expelled toward tube walls, and a structure resembling a meniscus is formed. This can be seen in Fig. 1(c). In such case, one can expect that the spin-imbalanced system separates into a spin-polarized core and a superfluid ring. Indeed, the unpaired particles occupy the less populated area and the vortex cores; see Fig. 5. One can also notice complex structures in the gas cloud formed by nodal lines. These are lines along which phase of the order parameter changes abruptly by $\pi$, and in Fig. 5 they are seen especially well in polarization $p(\boldsymbol{r})$ plots. While their presence may be a signature of the emergence of the FFLO-type phase, their complexity precludes stating that the resulting phase pattern can be approximated as a superposition of the vortex lattice phase and modulation of the sign along the radial direction. For example, in the cases $\delta=5 \%$ and $10 \%$ objects that emerge close to the tube center resemble recently predicted spin-polarized droplets [72] or soliton sacks [73] that are filled by vortices. In Fig. 5 their boundaries can be tracked by lines where the local polarization is nonzero. As we increase the imbalance above a threshold 


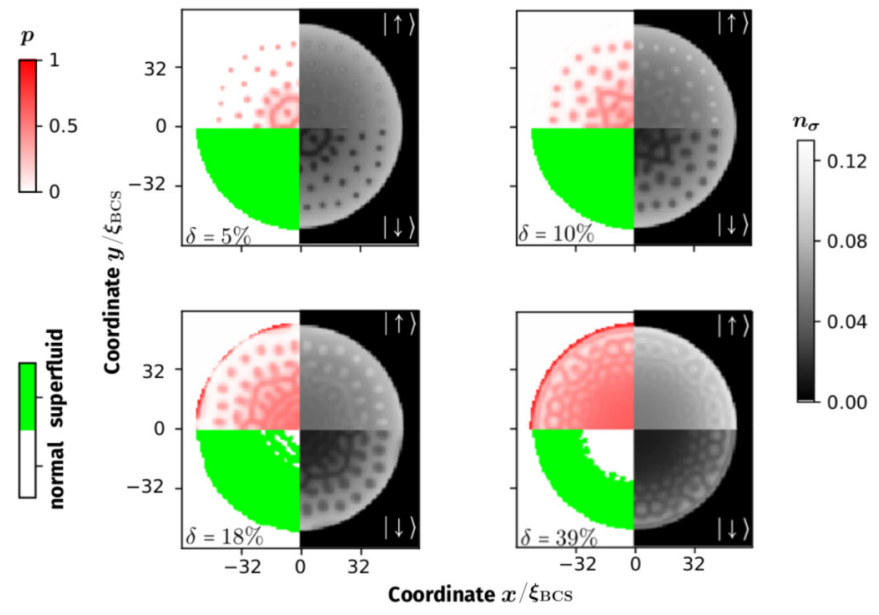

FIG. 5. Polarization $p(x, y, z=0)$ (top left part of each subplot), densities $n_{\sigma}(x, y, z=0)$ of the majority (top right part of each subplot), and the minority components (bottom right part of each subplot), as well as the indication of the superfluid part (bottom left part of each subplot).

value, in the studied case above $\delta \gtrsim 18 \%$, we start to destroy the superfluidity in the center. The system becomes phase separated, and we have a spin-polarized core in its center. The order parameter has a close to zero value there and nonzero in the superfluid ring with an oscillatory behavior on the interface. The superfluidity vanishes entirely when $\delta=62 \%$. The velocity profiles of the flow provide further indication that the phase separation process is dominant for relatively large population imbalances. We expect the spin-polarized (normal) part to rotate like a solid body with $v(r)=\Omega_{z} r$. The superfluid part can only support rotation via quantum vortices, and the velocity profile should be different, though. This is shown in Fig. 6. In the gas trapped within the harmonic potential, the core is superfluid, so the velocity in this region differs from the one corresponding to the solid-body rotation. As soon as we get to the corona, which is polarized, the profiles converge to expected $\Omega_{z} r$. On the other hand, the system in the box trap has a spin-polarized core and a superfluid outer part. The

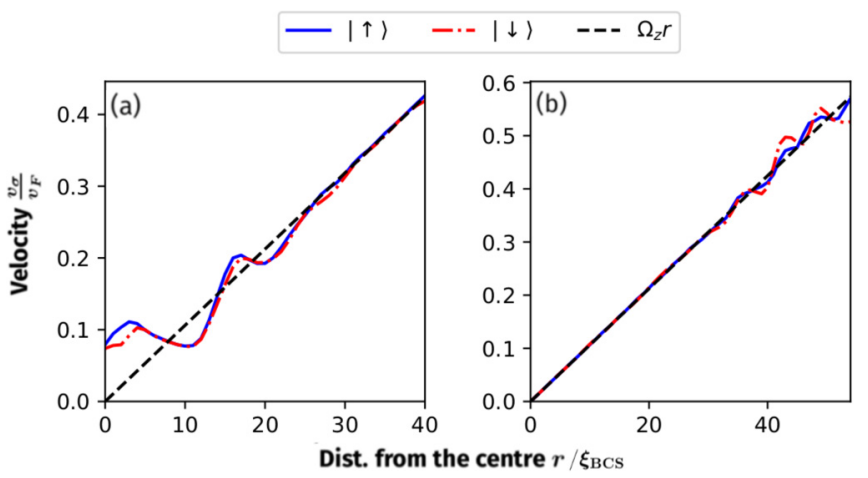

FIG. 6. Velocity profiles of the majority $|\uparrow\rangle$ (solid blue line) and minority $|\downarrow\rangle$ (dashed-dotted red line) components as functions of the distance from the system center $r$. Spin imbalance $\delta=39 \%$. Trapping potential: (a) the harmonic oscillator and (b) the tube. The dashed black line corresponds to the solid-body rotation.

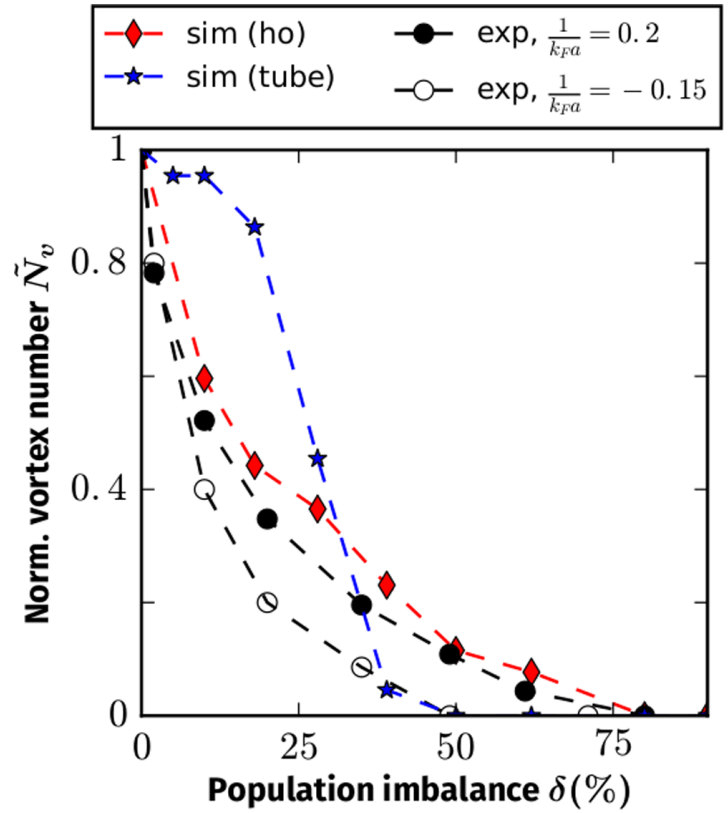

FIG. 7. Normalized number of vortices $\tilde{N}_{v} / N_{v \text {,max }}$ as a function of population imbalance $\delta$. Comparison between the experimental results (exp) for the Fermi gas on the BEC side $\left(\frac{1}{k_{F} a}=0.2\right)$, the BCS side $\left(\frac{1}{k_{F} a}=-0.15\right)$ near the unitary regime, and the simulation results (sim). Experimental points are taken from [20].

velocity profile near the core is linear, and it changes in the superfluid ring.

Finally, let us compare the global properties of the vortex lattice. In Fig. 7 we present the normalized vortex numbers defined as $\tilde{N}_{v}(\delta)=\frac{N_{v}(\delta)}{N_{v, \text { max }}}$ for different systems, including also experimental results for the harmonic trap. On a qualitative level, experimental and simulated numbers of vortices as a function of the population imbalance agree well. The fact that the data series from simulation is slightly above the experimental data does not necessarily mean that the DFT model is incorrect at the quantitative level. While in the simulation we can count the number of vortices accurately, in the experiment vortices close to the cloud boundary are gradually lost during the expansion [63]. That would lead to an underestimation of the number of vortices, especially for higher population imbalances, where they are not easily distinguishable due to the presence of unpaired particles in the vortex cores.

The change of the external potential vastly affects the vortex number as a function of the population imbalance; see the result for the box trap. There is a sharp decrease in $\tilde{N}_{v}$ between the values $\delta=18 \%$ and $39 \%$. Up to $\delta=18 \%$ almost the whole box is in a superfluid state, and above the spin-polarized region starts to develop in the center. At $\delta=$ $39 \%$, the remaining superfluid region close to the boundary becomes too small to accommodate any vortices.

\section{CONCLUSIONS}

The results presented in this paper demonstrate that the properties of rotating and spin-imbalance ultracold fermionic gas strongly depend on the control parameters: confining potential and population imbalance. The spatial separation of 
the system into superfluid and normal components appears as the leading process. The precise location of these components is determined by the shape of the confining potential. The normal component is composed mainly of the atoms being in the same spin state, and at the interference of the two phases proximity effects, typical for the superconductor-ferromagnet junction, emerge. They include sign oscillations of the order parameter, an effect regarded as a signature of the FFLO state. Rotation induces additional singularity points, around which the phase rotates by $2 \pi$, and the final pattern acquires a complex form. The presence of complex and nontriangular vortex patterns can be used as an indirect signature of the emergence of exotic superfluidity; however, this signal does not point directly to the FFLO phase. In the context of searching for the FFLO state, it is desired to minimize the number of inhomogeneity sources and search for well-developed modulations of the order parameter over large scales, compared to coherence length.

\section{ACKNOWLEDGMENTS}

We thank Martin Zwierlein for the helpful insight into the experiment. We also thank Piotr Magierski for reading a draft of the manuscript and for valuable criticism. This work was supported by the Polish National Science Center (NCN) under Contract No. UMO-2017/26/E/ST3/00428. In the final stage of work, J.K. was supported under Contract No. UMO-2019/34/E/ST2/00289. We acknowledge the Interdisciplinary Centre for Mathematical and Computational Modelling (ICM) of Warsaw University for computing resources at Okeanos (Grant No. GA83-9) and PL-Grid Infrastructure for providing us resources at the Prometheus supercomputer. The Center for Theoretical Physics of the Polish Academy of Sciences is a member of the $\mathrm{Na}$ tional Laboratory of Atomic, Molecular, and Optical Physics (KL FAMO).
[1] J. R. Abo-Shaeer, C. Raman, J. M. Vogels, and W. Ketterle, Observation of vortex lattices in Bose-Einstein condensates, Science 292, 476 (2001).

[2] M. W. Zwierlein, J. R. Abo-Shaeer, A. Schirotzek, C. H. Schunck, and W. Ketterle, Vortices and superfluidity in a strongly interacting Fermi gas, Nature (London) 435, 1047 (2005).

[3] M. Takahashi, T. Mizushima, M. Ichioka, and K. Machida, Vortex-Core Structure in Neutral Fermion Superfluids with Population Imbalance, Phys. Rev. Lett. 97, 180407 (2006).

[4] Y. P. Shim, R. A. Duine, and A. H. MacDonald, Fulde-FerrellLarkin-Ovchinnikov vortex lattice states in fermionic cold-atom systems, Phys. Rev. A 74, 053602 (2006).

[5] I. Bausmerth, A. Recati, and S. Stringari, Unitary polarized Fermi gas under adiabatic rotation, Phys. Rev. A 78, 063603 (2008).

[6] M. L. Kulić, A. Sedrakian, and D. H. Rischke, Rotating Larkin-Ovchinnikov-Fulde-Ferrell state of the two-dimensional ultracold Fermi superfluid gas: Reentrant behavior of the critical angular velocity, Phys. Rev. A 80, 043610 (2009).

[7] X. L. Shang, P. M. Zhang, and W. Zuo, The single vortex in the FFLO state, Phys. Lett. A 374, 1866 (2010).

[8] H. J. Warringa, Location of the vortex phase in the phase diagram of a rotating two-component Fermi gas, Phys. Rev. A 86, 043615 (2012).

[9] K. B. Gubbels and H. T. C. Stoof, Imbalanced Fermi gases at unitarity, Phys. Rep. 525, 255 (2013).

[10] L. Radzihovsky and D. E. Sheehy, Imbalanced Feshbachresonant Fermi gases, Rep. Prog. Phys. 73, 076501 (2010).

[11] J. J. Kinnunen, J. E. Baarsma, J. P. Martikainen, and P. Törmä, The Fulde-Ferrell-Larkin-Ovchinnikov state for ultracold fermions in lattice and harmonic potentials: A review, Rep. Prog. Phys. 81, 046401 (2018).

[12] G. B. Partridge, Pairing and phase separation in a polarized Fermi gas, Science 311, 503 (2006).

[13] Y. Shin, M. W. Zwierlein, C. H. Schunck, A. Schirotzek, and W. Ketterle, Observation of Phase Separation in a Strongly
Interacting Imbalanced Fermi Gas, Phys. Rev. Lett. 97, 030401 (2006).

[14] Y. Liao, A. S. C. Rittner, T. Paprotta, W. Li, G. B. Partridge, R. G. Hulet, S. K. Baur, and E. J. Mueller, Spin-imbalance in a one-dimensional Fermi gas, Nature (London) 467, 567 (2010).

[15] D. H. Kim, J. J. Kinnunen, J. P. Martikainen, and P. Törmä, Exotic Superfluid States of Lattice Fermions in Elongated Traps, Phys. Rev. Lett. 106, 095301 (2011).

[16] B. A. Olsen, M. C. Revelle, J. A. Fry, D. E. Sheehy, and R. G. Hulet, Phase diagram of a strongly interacting spin-imbalanced Fermi gas, Phys. Rev. A 92, 063616 (2015).

[17] D. Inotani, S. Yasui, T. Mizushima, and M. Nitta, Radial Fulde-Ferrell-Larkin-Ovchinnikov-like state in a populationimbalanced Fermi gas, Phys. Rev. A 103, 053308 (2021).

[18] H. Hu, X.-J. Liu, and P. D. Drummond, Visualization of Vortex Bound States in Polarized Fermi Gases at Unitarity, Phys. Rev. Lett. 98, 060406 (2007).

[19] P. Magierski, G. Wlazłowski, A. Makowski, and K. Kobuszewski, Spin-polarized vortices with reversed circulation, arXiv:2011.13021.

[20] M. W. Zwierlein, A. Schirotzek, C. H. Schunck, and W. Ketterle, Fermionic superfluidity with imbalanced spin populations, Science 311, 492 (2006).

[21] M. Croitoru and A. Buzdin, In search of unambiguous evidence of the Fulde-Ferrell-Larkin-Ovchinnikov state in quasi-lowdimensional superconductors, Condens. Matter 2, 30 (2017).

[22] T. Mizushima, K. Machida, and M. Ichioka, Topological Structure of a Vortex in the Fulde-Ferrell-Larkin-Ovchinnikov State, Phys. Rev. Lett. 95, 117003 (2005).

[23] M. Ichioka and K. Machida, Vortex states in superconductors with strong Pauli-paramagnetic effect, Phys. Rev. B 76, 064502 (2007).

[24] M. Ichioka, H. Adachi, T. Mizushima, and K. Machida, Vortex state in a Fulde-Ferrell-Larkin-Ovchinnikov superconductor based on quasiclassical theory, Phys. Rev. B 76, 014503 (2007).

[25] H. Shimahara, Transition from the vortex state to the FuldeFerrell-Larkin-Ovchinnikov state in quasi-two-dimensional superconductors, Phys. Rev. B 80, 214512 (2009). 
[26] T. Yokoyama, M. Ichioka, and Y. Tanaka, Theory of pairing symmetry in Fulde-Ferrell-Larkin-Ovchinnikov vortex state and vortex lattice, J. Phys. Soc. Jpn. 79, 034702 (2010).

[27] H. Shimahara and D. Rainer, Crossover from vortex states to the Fulde-Ferrell-Larkin-Ovchinnikov state in two-dimensional $s$ - and $d$-wave superconductors, J. Phys. Soc. Jpn. 66, 3591 (1997).

[28] U. Klein, D. Rainer, and H. Shimahara, Interplay of Fulde-Ferrell-Larkin-Ovchinnikov and vortex states in twodimensional superconductors, J. Low Temp. Phys. 118, 91 (2000).

[29] K. Yang and A. H. MacDonald, Vortex-lattice structure of Fulde-Ferrell-Larkin-Ovchinnikov superconductors, Phys. Rev. B 70, 094512 (2004).

[30] U. Klein, Two-dimensional superconductor in a tilted magnetic field: States with finite Cooper-pair momentum, Phys. Rev. B 69, 134518 (2004).

[31] S. Sugiura, T. Isono, T. Terashima, S. Yasuzuka, J. A. Schlueter, and S. Uji, Fulde-Ferrell-Larkin-Ovchinnikov and vortex phases in a layered organic superconductor, npj Quantum Mater. 4, 7 (2019).

[32] K. M. Suzuki, K. Machida, Y. Tsutsumi, and M. Ichioka, Microscopic Eilenberger theory of Fulde-Ferrell-Larkin-Ovchinnikov states in the presence of vortices, Phys. Rev. B 101, 214516 (2020).

[33] A. Bulgac, M. M. Forbes, and P. Magierski, The unitary Fermi gas: From Monte Carlo to density functionals, in The BCS-BEC Crossover and the Unitary Fermi Gas, edited by Wilhelm Zwerger (Springer, Berlin, 2012), pp. 305-373.

[34] A. Bulgac and Y. Yu, Vortex State in a Strongly Coupled Dilute Atomic Fermionic Superfluid, Phys. Rev. Lett. 91, 190404 (2003).

[35] A. Bulgac, Y.-L. Luo, P. Magierski, K. J. Roche, and Y. Yu, Real-time dynamics of quantized vortices in a unitary Fermi superfluid, Science 332, 1288 (2011).

[36] A. Bulgac, M. M. Forbes, M. M. Kelley, K. J. Roche, and G. Wlazłowski, Quantized Superfluid Vortex Rings in the Unitary Fermi Gas, Phys. Rev. Lett. 112, 025301 (2014).

[37] G. Wlazłowski, A. Bulgac, M. M. Forbes, and K. J. Roche, Life cycle of superfluid vortices and quantum turbulence in the unitary Fermi gas, Phys. Rev. A 91, 031602(R) (2015).

[38] G. Wlazłowski, K. Sekizawa, M. Marchwiany, and P. Magierski, Suppressed Solitonic Cascade in Spin-Imbalanced Superfluid Fermi Gas, Phys. Rev. Lett. 120, 253002 (2018).

[39] A. Bulgac and M. M. Forbes, Unitary Fermi Supersolid: The Larkin-Ovchinnikov Phase, Phys. Rev. Lett. 101, 215301 (2008).

[40] S. Simonucci, P. Pieri, and G. C. Strinati, Vortex arrays in neutral trapped Fermi gases through the BCS-BEC crossover, Nat. Phys. 11, 941 (2015).

[41] P. Pieri and G. C. Strinati, Trapped Fermions with Density Imbalance in the Bose-Einstein Condensate Limit, Phys. Rev. Lett. 96, 150404 (2006).

[42] R. Turolla, S. Zane, and A. L. Watts, Magnetars: The physics behind observations. A review, Rep. Prog. Phys. 78, 116901 (2015).

[43] D. Blaschke and N. Chamel, Phases of dense matter in compact stars, in The Physics and Astrophysics of Neutron Stars (Springer International Publishing, Cham, Switzerland, 2018), pp. 337400.
[44] G. Baym, C. Pethick, and D. Pines, Superfluidity in neutron stars, Nature (London) 224, 673 (1969).

[45] P. W. Anderson and N. Itoh, Pulsar glitches and restlessness as a hard superfluidity phenomenon, Nature (London) 256, 25 (1975).

[46] D. Pines and M. A. Alpar, Superfluidity in neutron stars, Nature (London) 316, 27 (1985).

[47] B. Haskell and A. Melatos, Models of pulsar glitches, Int. J. Mod. Phys. D 24, 1530008 (2015).

[48] M. Stein, X.-G. Huang, A. Sedrakian, and J. W. Clark, Phase diagram of dilute nuclear matter: Unconventional pairing and the BCS-BEC crossover, Phys. Rev. C 86, 062801(R) (2012).

[49] M. Stein, A. Sedrakian, X.-G. Huang, and J. W. Clark, BCS-BEC crossovers and unconventional phases in dilute nuclear matter, Phys. Rev. C 90, 065804 (2014).

[50] M. Stein, A. Sedrakian, X.-G. Huang, and J. W. Clark, Spinpolarized neutron matter: Critical unpairing and BCS-BEC precursor, Phys. Rev. C 93, 015802 (2016).

[51] M. Bender, P.-H. Heenen, and P.-G. Reinhard, Self-consistent mean-field models for nuclear structure, Rev. Mod. Phys. 75, 121 (2003).

[52] A. Bulgac, Time-dependent density functional theory for fermionic superfluids: From cold atomic gases to nuclei and neutron star crust, Phys. Status Solidi B 256, 1800592 (2019).

[53] G. Colò, Nuclear density functional theory, Adv. Phys.: X 5, 1740061 (2020).

[54] A. Bulgac, Local density approximation for systems with pairing correlations, Phys. Rev. C 65, 051305(R) (2002).

[55] A. Baran, A. Bulgac, M. M. Forbes, G. Hagen, W. Nazarewicz, N. Schunck, and M. V. Stoitsov, Broyden's method in nuclear structure calculations, Phys. Rev. C 78, 014318 (2008).

[56] D. D. Johnson, Modified Broyden's method for accelerating convergence in self-consistent calculations, Phys. Rev. B 38, 12807 (1988).

[57] W-SLDA Toolkit, https://wslda.fizyka.pw.edu.pl/.

[58] S. Nascimbène, N. Navon, K. J. Jiang, L. Tarruell, M. Teichmann, J. McKeever, F. Chevy, and C. Salomon, Collective Oscillations of an Imbalanced Fermi Gas: Axial Compression Modes and Polaron Effective Mass, Phys. Rev. Lett. 103, 170402 (2009).

[59] N. Navon, S. Nascimbene, F. Chevy, and C. Salomon, The equation of state of a low-temperature Fermi gas with tunable interactions, Science 328, 729 (2010).

[60] J. C. Pei, J. Dukelsky, and W. Nazarewicz, Competition between normal superfluidity and Larkin-Ovchinnikov phases of polarized Fermi gases in elongated traps, Phys. Rev. A 82, 021603(R) (2010).

[61] A. I. Buzdin, Proximity effects in superconductor-ferromagnet heterostructures, Rev. Mod. Phys. 77, 935 (2005).

[62] S. Matyjaśkiewicz, M. H. Szymańska, and K. Góral, Probing Fermionic Condensates by Fast-Sweep Projection onto Feshbach Molecules, Phys. Rev. Lett. 101, 150410 (2008).

[63] C. H. Schunck, M. W. Zwierlein, A. Schirotzek, and W. Ketterle, Superfluid Expansion of a Rotating Fermi Gas, Phys. Rev. Lett. 98, 050404 (2007). 
[64] Y. Shin, C. H. Schunck, A. Schirotzek, and W. Ketterle, Phase diagram of a two-component Fermi gas with resonant interactions, Nature (London) 451, 689 (2008).

[65] M. H. Szymańska, B. D. Simons, and K. Burnett, Dynamics of the BCS-BEC Crossover in a Degenerate Fermi Gas, Phys. Rev. Lett. 94, 170402 (2005).

[66] S. Simonucci, P. Pieri, and G. C. Strinati, Bound states in a superfluid vortex: A detailed study along the BCS-BEC crossover, Phys. Rev. B 99, 134506 (2019).

[67] A. L. Gaunt, T. F. Schmidutz, I. Gotlibovych, R. P. Smith, and Z. Hadzibabic, Bose-Einstein Condensation of Atoms in a Uniform Potential, Phys. Rev. Lett. 110, 200406 (2013).

[68] L. Chomaz, L. Corman, T. Bienaimé, R. Desbuquois, C. Weitenberg, S. Nascimbène, J. Beugnon, and J. Dalibard, Emergence of coherence via transverse condensation in a uniform quasi-two-dimensional Bose gas, Nat. Commun. 6, 6162 (2015).

[69] N. Navon, A. L. Gaunt, R. P. Smith, and Z. Hadzibabic, Emergence of a turbulent cascade in a quantum gas, Nature (London) 539, 72 (2016).

[70] B. Mukherjee, Z. Yan, P. B. Patel, Z. Hadzibabic, T. Yefsah, J. Struck, and M. W. Zwierlein, Homogeneous Atomic Fermi Gases, Phys. Rev. Lett. 118, 123401 (2017).

[71] N. Navon, R. P. Smith, and Z. Hadzibabic, Quantum gases in optical boxes, arXiv:2106.09716.

[72] P. Magierski, B. Tüzemen, and G. Wlazłowski, Spin-polarized droplets in the unitary Fermi gas, Phys. Rev. A 100, 033613 (2019).

[73] M. Barkman, A. Samoilenka, T. Winyard, and E. Babaev, Ring solitons and soliton sacks in imbalanced fermionic systems, Phys. Rev. Res. 2, 043282 (2020). 